\title{
DOS EDIFICIOS PUBLICOS A BASE DE TIERRA EN CHILE
}

\author{
(TWO PUBLIC BUILDINGS BASICALLY MADE OF EARTH IN CHILE)
}

Hugo Pereira, Arquitecto

RESUMEN

Se presentan dos realizaciones en Chile: la Casa de la Cultura, en la barriada La Florida (Santiago) y la Escuela Concentrada de Botalcura, en la VIII Región. Ambas construcciones tienen en común el haberse realizado a base de adobe y cerchas de madera, el presentar una cierta continuidad en los criterios de diseño adoptados, en ambas ha participado el Arquitecto Hugo Pereira, y ser edificios recién construidos, incluso parcialmente en construcción.

El hecho de tratarse de espacios con luces y alturas propias de construcciones para uso público, asi como la circunstancia de que la parte construida de la Casa de la Cultura en el momento del último gran sismo en Chile (marzo 1985), presentase un excelente comportamiento, son hechos que hacen especialmente interesante la publicación de estas realizaciones.

\section{SUMMARY}

Two works in Chile are reported: The House of the Culture in La Florida (Santiago) and the Botalcura Concentred School in the VIIlth Zone. Both constructions have as common

characteristic to be built with adobe and wooden girders, to present a certain continuity in the adapted criteria of desing -both from Architect Hugo Pereira- and to be newly constructed buildings, even partialy in construction.

The fact that there are spaces with appropriate light and height for public use, so as the circumstance that the part which waas being constructed just at the moment of the great seism in Chile (February 1985) had and excelent behaviour, makes these works interesting to be published.

\section{CASA DE LA CULTURA DE LA I. MUNICIPALIDAD DE LA FLORIDA, SANTIAGO-CHILE}

\begin{tabular}{|c|c|c|}
\hline 1. & $\begin{array}{l}\text { Alcalde } \\
\text { Jefe POJH }\end{array}$ & $\begin{array}{l}\text { Sr. Walton Ojeda V. } \\
\text { Ing. Sr. Gilles Galte L. }\end{array}$ \\
\hline \multirow[t]{2}{*}{2.} & $\begin{array}{l}\text { Arquitecto } \\
\text { Directivo }\end{array}$ & Sr. Hugo Pereira G. \\
\hline & Jefe Proyecto & Arqto. Sr. Pabio Astaburuaga G. \\
\hline \multirow[t]{4}{*}{3.} & $\begin{array}{l}\text { Supervisores de } \\
\text { Obra }\end{array}$ & $\begin{array}{l}\text { Sr. E. Campos. } \\
\text { Sr. C. Riquelme. } \\
\text { Sr. C. Bustamante. }\end{array}$ \\
\hline & Asesoria Calculo & Ing. Sr. Pedro Astaburuaga G. \\
\hline & Asesoria Técnica & $\begin{array}{l}\text { C. C. Sr. Gastón Barrios L. Esc. Construcción Civil, Universidad } \\
\text { Católica de Chile. }\end{array}$ \\
\hline & $\begin{array}{l}\text { Taller Profesional } \\
\text { Proyectos POJH }\end{array}$ & $\begin{array}{l}\text { Arqtos. M. Cuiza, G. Garcia, R. Meschi, J. Morales, M. Rodri- } \\
\text { guez, J. Topaz. }\end{array}$ \\
\hline
\end{tabular}


Este proyecto, ubicado en una de las Comunas dormitorio de Santiago, sector habitacional caracterizado por una población de pequeños empleados y algunos sectores de marginalidad, viene a constituirse y a reforzar un área que ya cuenta con cierto equipamiento comunitario. Funcionan en ella la piscina municipal, la Sede de la Corporación Municipal de Educación y se construye un Gimnasio techado. La idea de este proyecto nace determinada bajo tres condicionantes: Primero, durante el año 1983, los niveles de cesantia en el pais alcanzan niveles muy altos (cerca del $30 \%$ ), el Gobierno implementa un Plan Ocupacional de Jefes de Hogar (POJH). Segunda, la mano de obra de los adscritos a dicho plan, en su gran mayoria constituida por sectores muy jóvenes (entre 18 y 25 años), no tenian capacitación técnica en labores de construcción. Tercero, la estructura económica del POJH permitia invertir en materiales, para edificar, solamente un $25 \%$ del valor global de los proyectos. Es asi, como, agregando la urgente necesidad, a la fecha de implementar planes masivos de absorción de mano de obra, diseñé este proyecto, el cual fue desarrollado en la Oficina de Profesionales del POJH durante el año 1983 y se construye por etapas, en 1984 y 1985.

El proyecto se elabora, eligiendo una antigua tradición del campo chileno: la construcción de

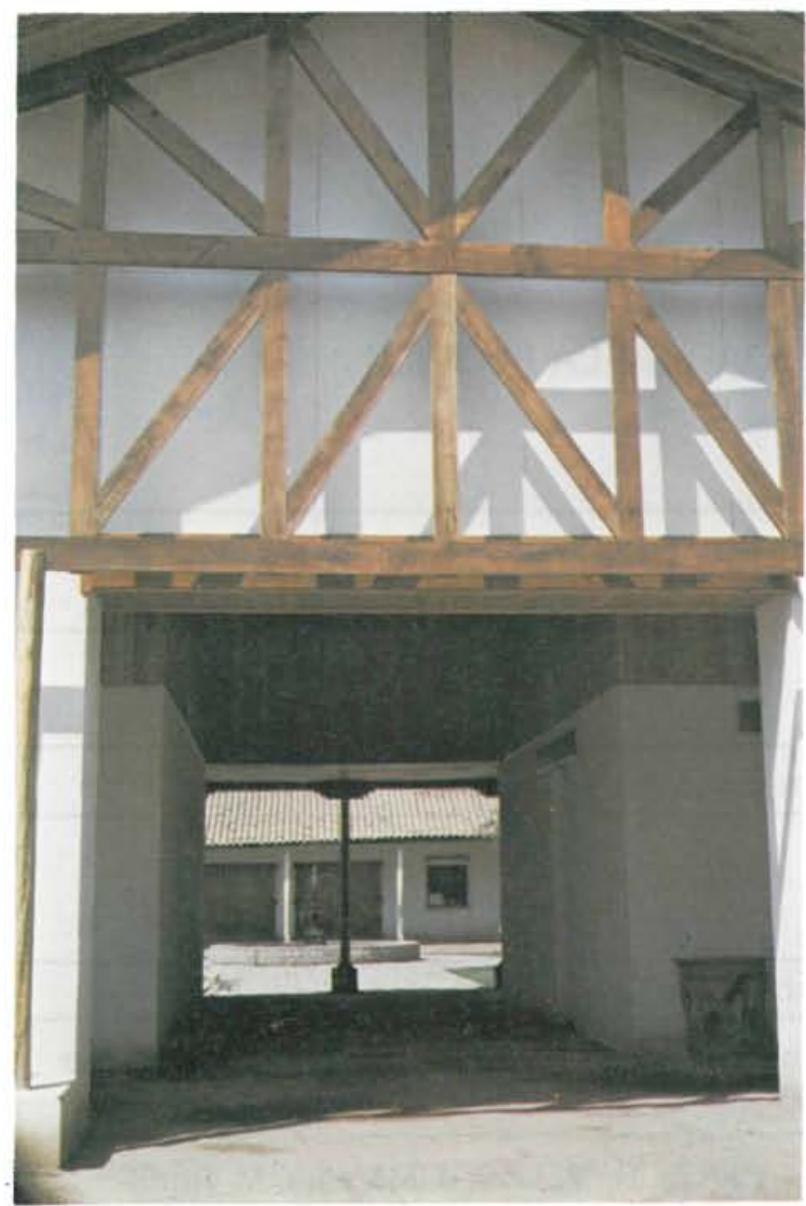

Fig. 1.-Detalle de la entrada principal. adobe. Material abundante en la zona del Valle Central de Chile y muy apropiado para sus condicionantes climatológicas y de uso social. Se refuerza la imagen de la antigua casa patronal, existente en el terreno, la que habia sido restaurada recientemente por la Municipalidad. Tenia esta casona, aproximadamente, $1.300 \mathrm{~m}^{2}$ edificados. Con el nuevo proyecto se amplia esta capacidad a una superficie total de $2.859 \mathrm{~m}^{2}$.

El nuevo proyecto contempla básicamente cuatro áreas: Administrativa, Biblioteca, Auditorium (300 personas), Talleres Artesanales y Museo. A través de la edificación propuesta se busca la valorización de una "identidad cultural" propia del pais, rescatando el patrimonio que constituyen las casas de campo, construidas en Chile desde el siglo XVI hasta hoy dia.

El sistema constructivo elegido, fue el de albañileria de adobe, de cabeza, de $10 \times 30 \times 58 \mathrm{~cm}$. con una altura máxima de muros de $3 \mathrm{~m}$. Cerchas de pino $(2 \times 6)$ y cubierta de teja de arcilla cocida. En obra se instalaron una fábrica de adobes y una fábrica de tejas, alcanzándose una producción máxima semanal de 2.000 y 4.000 respectivamente. La resistencia a la compresión exigida al adobe y controlada en laboratorio de ensayes era de $16 \mathrm{~kg} / \mathrm{cm}^{2}$. Para la confección de éstos se utilizó tierra de demolición de construc-

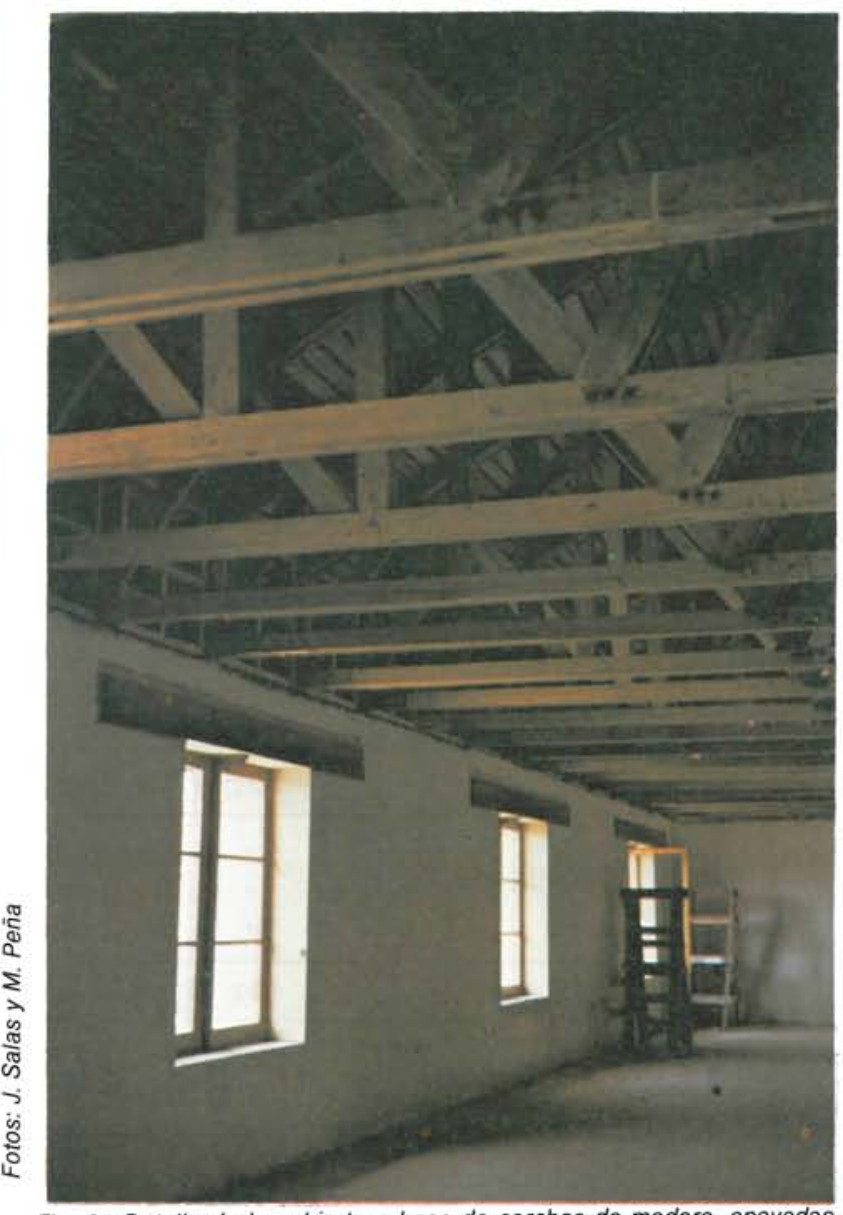

Fig. 2.-Detalle de la cubierta a base de cerchas de madera, apoyadas sobre muros de adobe. 
ciones de adobe aledañas al sector de la obra, lo que mejora la resistencia de los adobes ya que hay una mayor presencia de fibras. Al material de tierra se le agregó paja de trigo, en una proporción de $5 \mathrm{~kg} / \mathrm{m}^{3}$. Se utilizaron adoberas de madera de rauli. Las tejas fabricadas fueron del tipo "colonial" o "árabe" con forma de canal tronco-cónica y a diferencia de lo utilizado en la arquitectura tradicional, éstas fueron amarradas a la cubierta mediante alambre galvanizado, dado que en situaciones sismicas, las tejas constituyen un gran peligro para los moradores.

Es interesante constatar que, empleando esta sencilla técnica, y aplicando un buen control de calidad en obra, coordinado con una asesoria técnica creativa, es posible llegar a óptimos resultados. Prueba de esto, es que este edificio soportó en excelentes condiciones el terremoto del 3 de marzo de 1985, en circunstancias que la estructura de techumbre estaba parcialmente ejecutada.
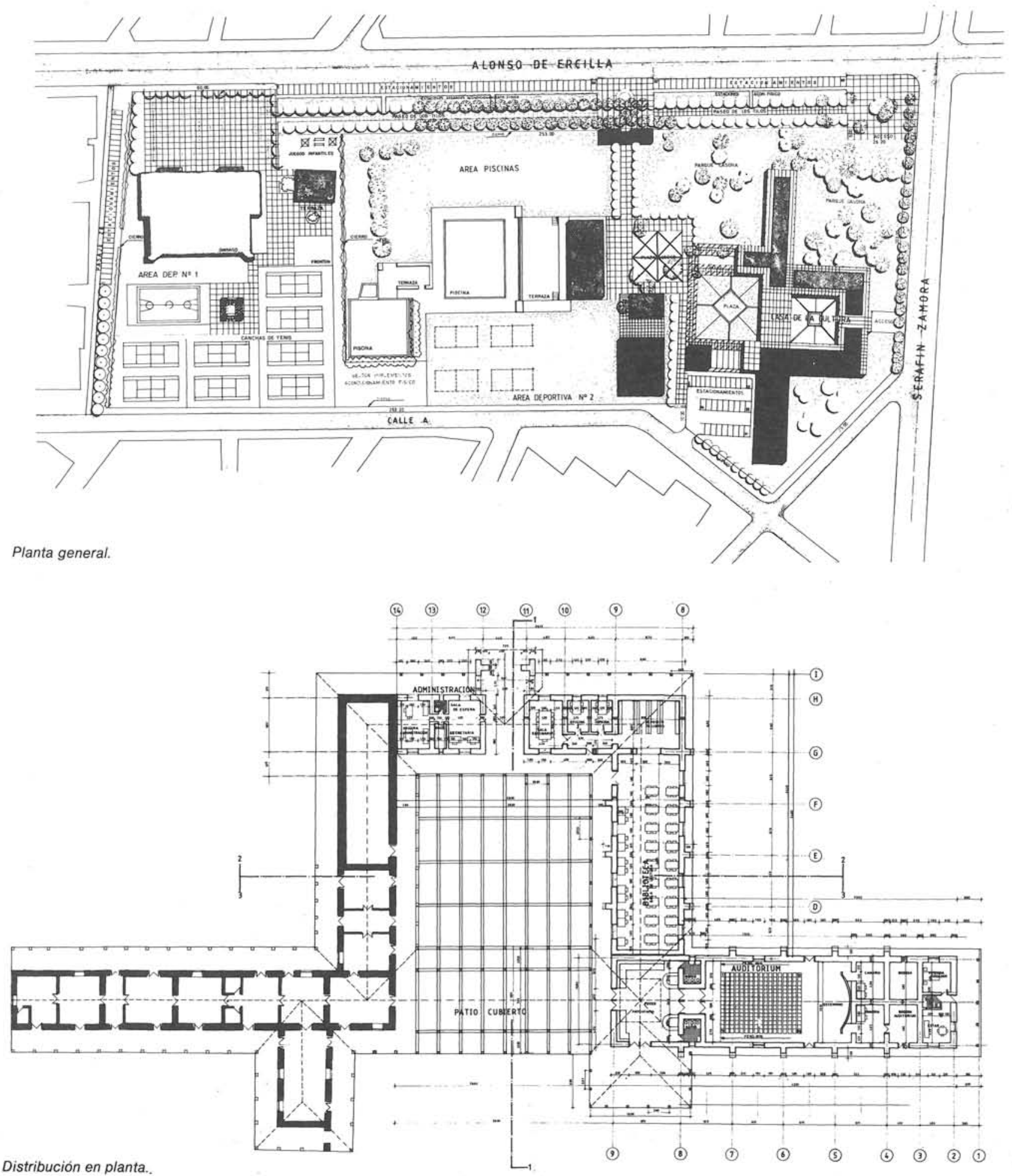


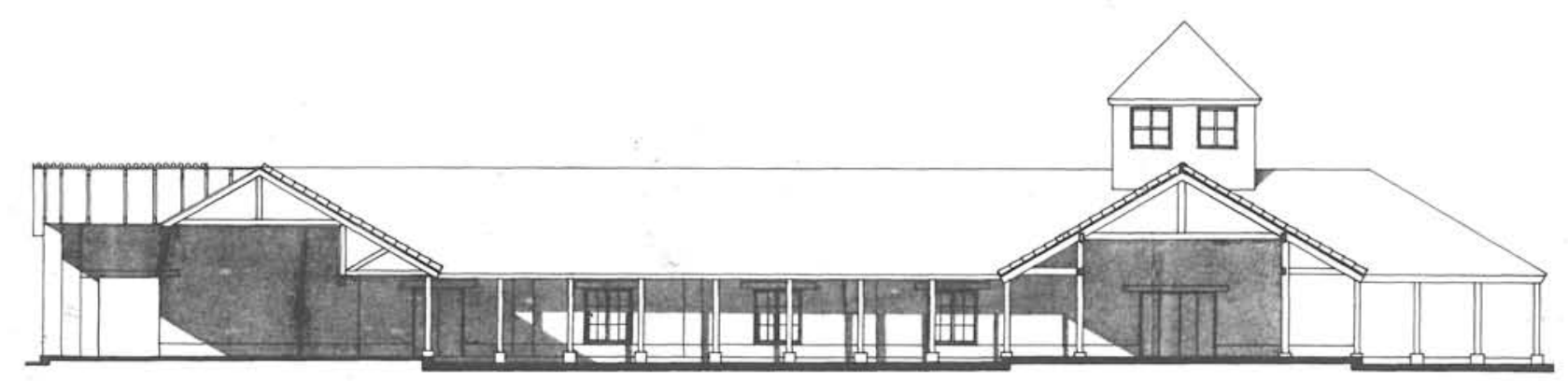

Seccion-Alzado 1-1.

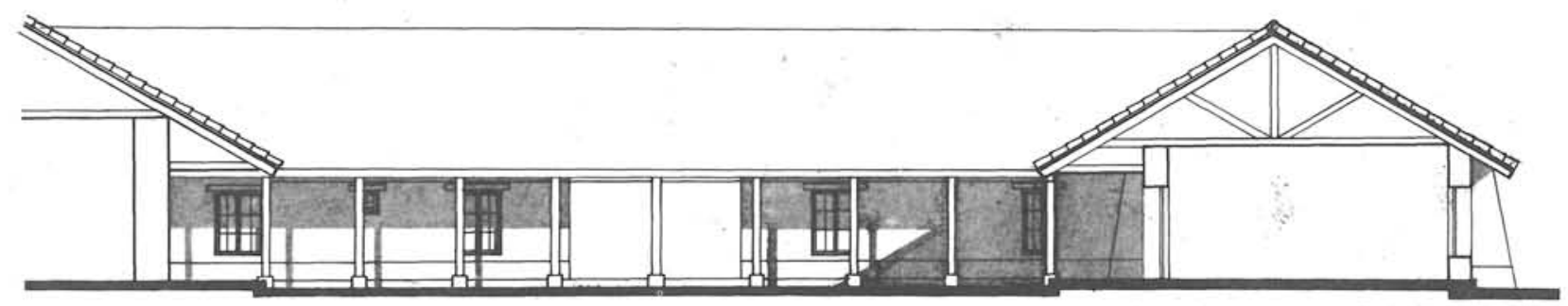

Seccion-Alzado 2-2.

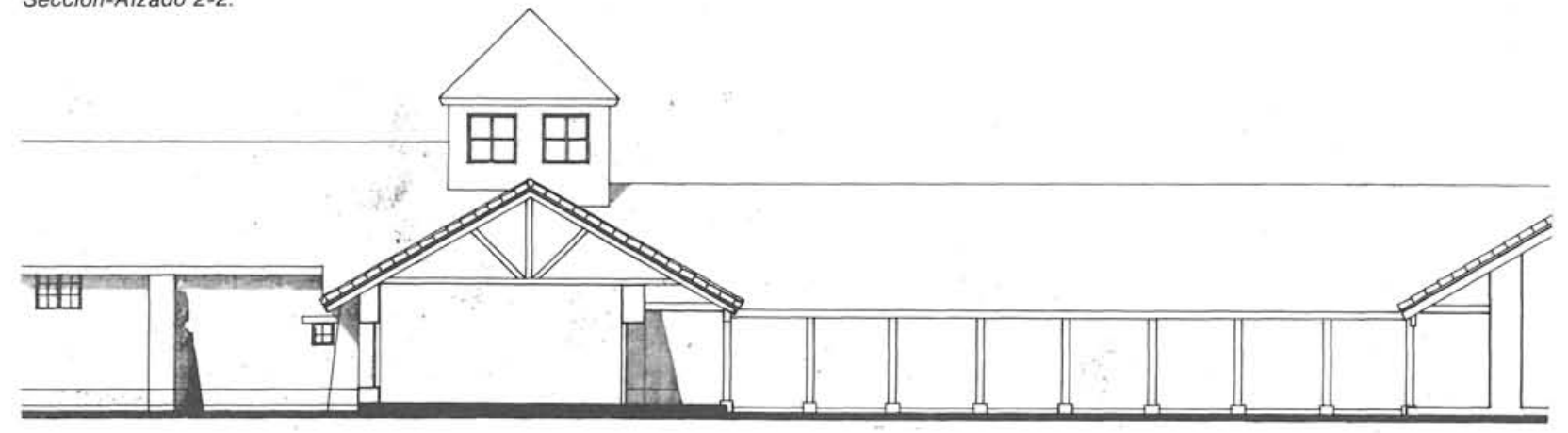

Seccion-Alzado 3-3.

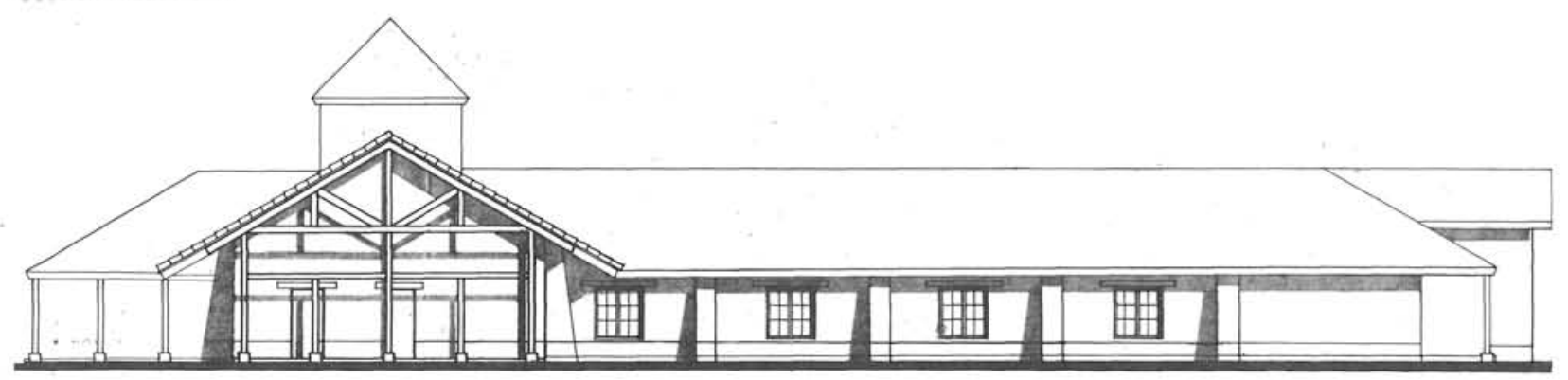

Alzado Este.

田田

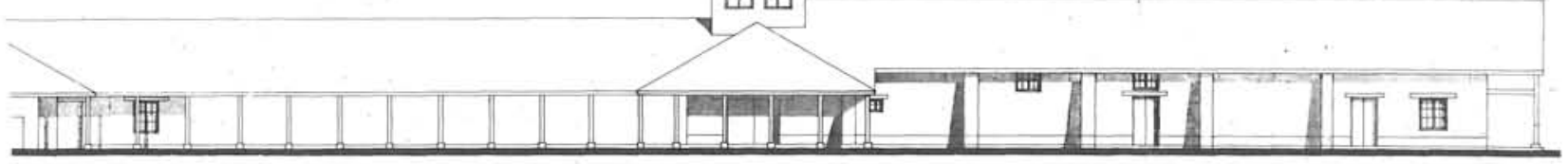

Alzado Sur.

田田

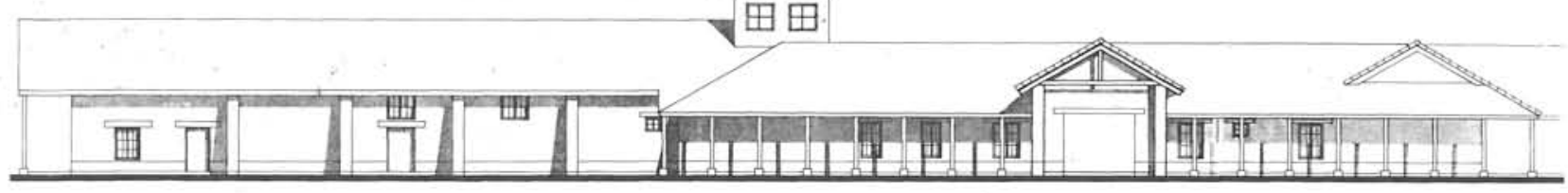

Alzado Norte. 


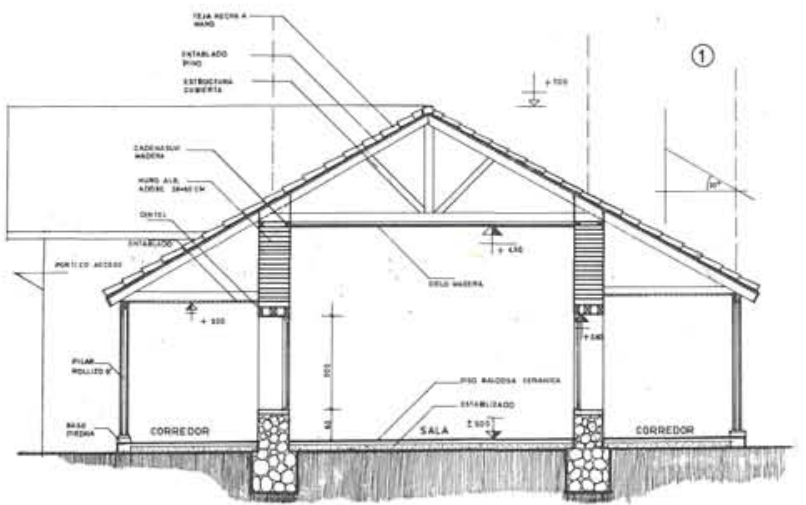

Seccion Administracion.

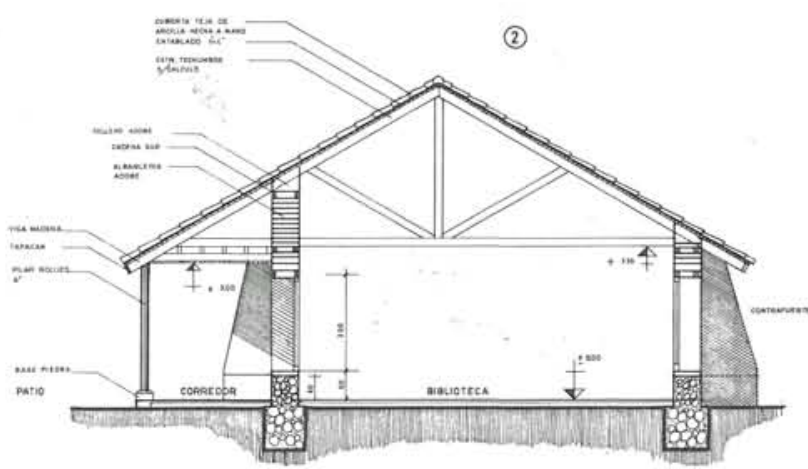

Sección Biblioteca.

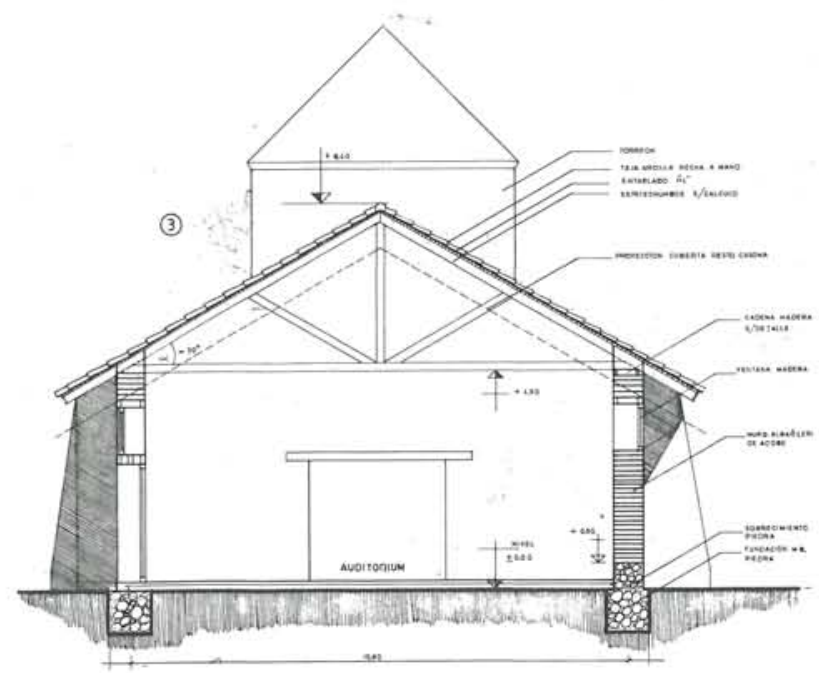

Sección Auditorio.

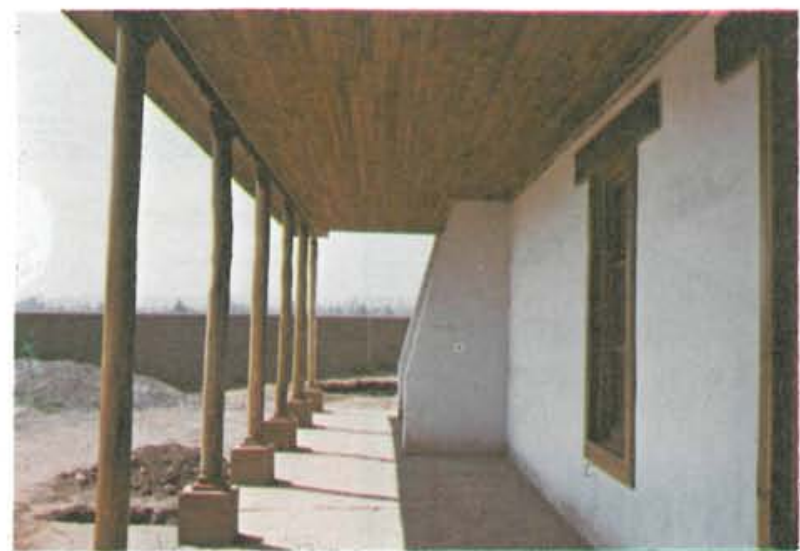

Fig. 3.-Detalle del porche y contrafuerte.

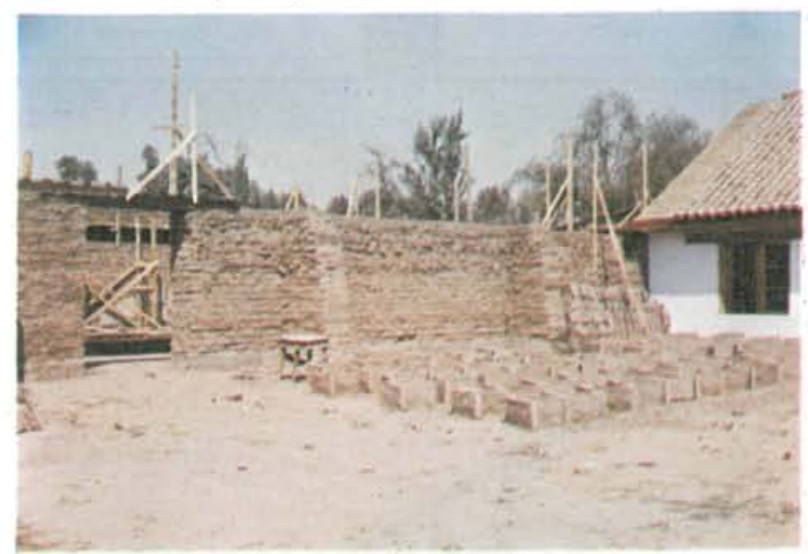

Fig. 4. - Zona en construccion y secado de adobes.

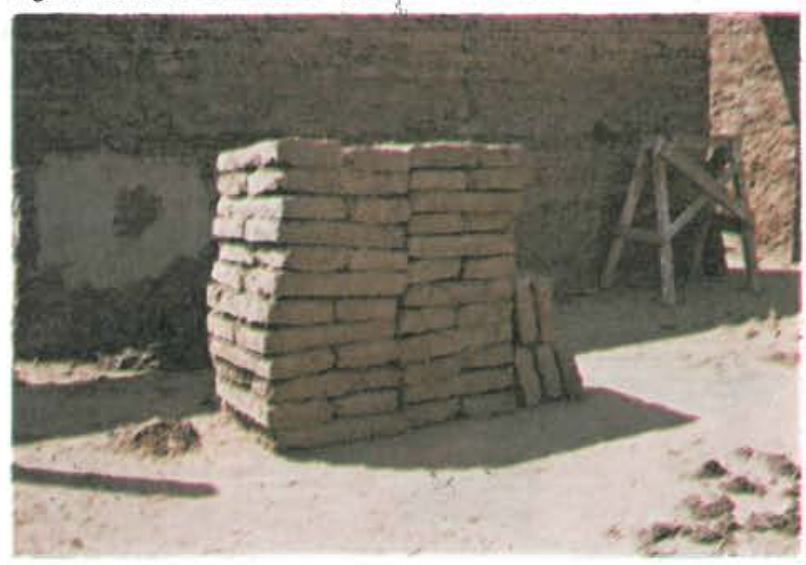

Fig. 5.-Apilado de adobes en obra.

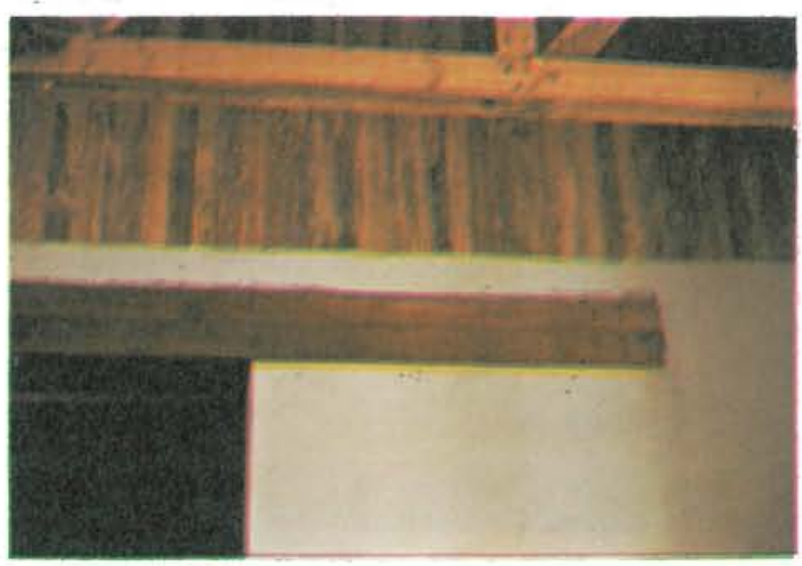

Fig 6.-Detalle del empotramiento del dintel. 


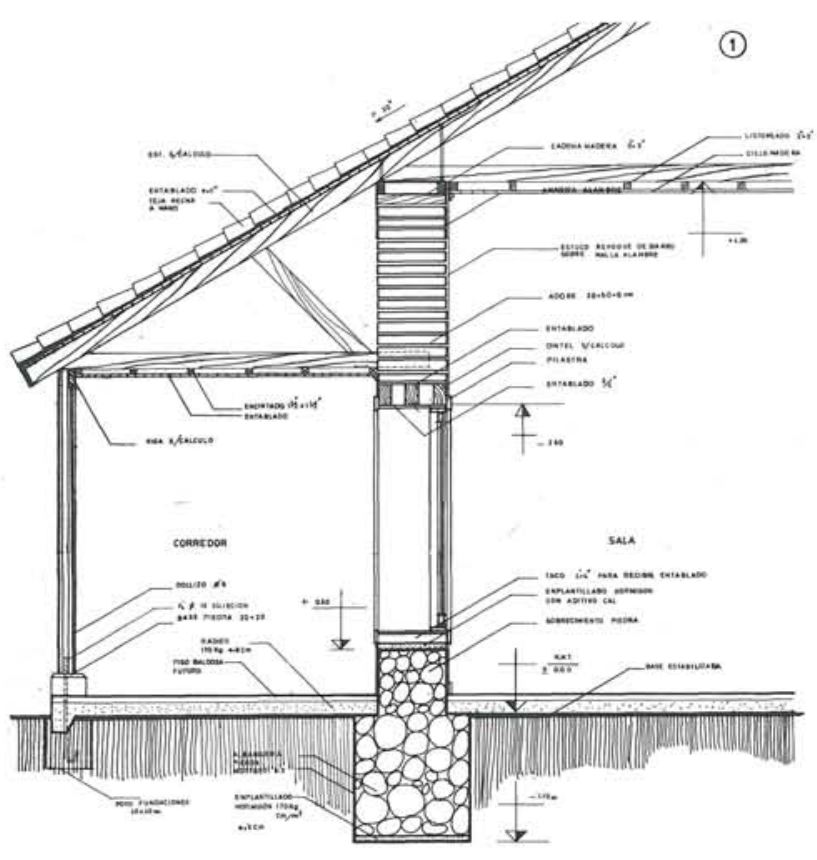

Escantillones

(detalles constructivos 1,2 y 3 ).
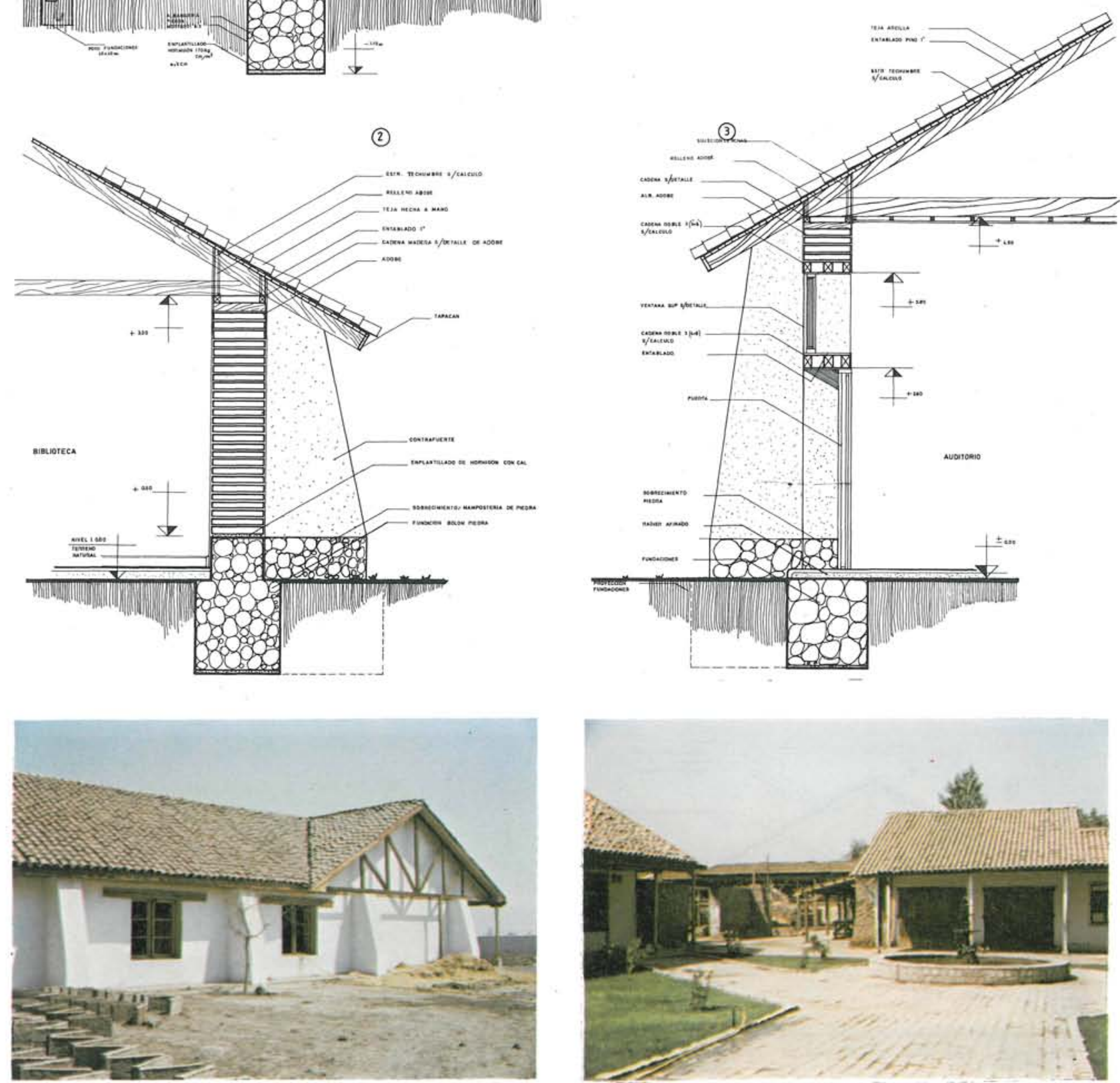

Fig. 7.- Vista lateral de una de las alas construidas.

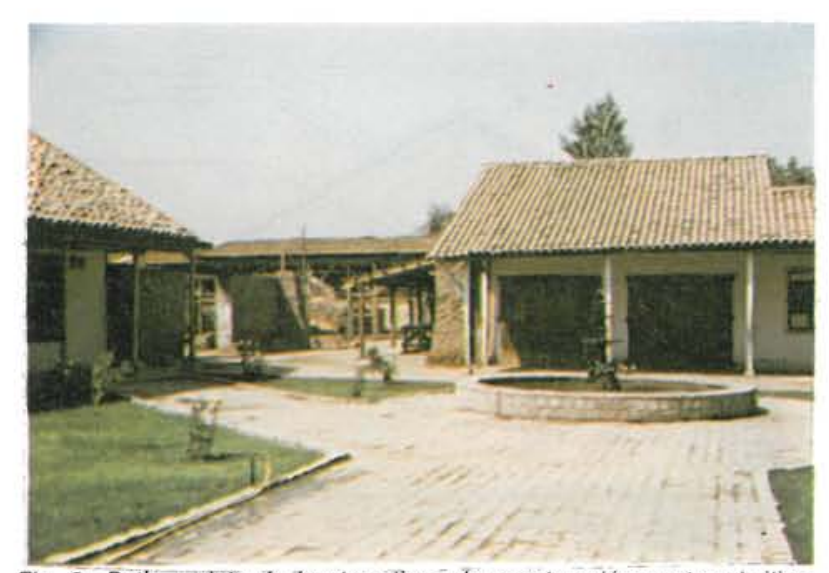

Fig. 8.-Patio y vista de los tres tipos de construcción: parte primitiva, ejecutada hace dos años y en construcción. 


\section{ESCUELA CONCENTRADA DE BOTALCURA: VIII REGION - CHILE}

La Comuna de Pencahue, con una superficie de $1.600 \mathrm{Km}^{2}$ y una población de 7.744 habitantes, ubicada en pleno Valle Central chileno, recoge la tradición campesiona del adobe. Es una Comuna de evidente carácter agricola - ganadero, siendo sus principales productos, los viñedos, las hortalizas y el oro. Los principales problemas derivan del aislamiento de muchos sectores de dificil accesibilidad, especialmente en lo que dice relación con la actividad educacional. De aqui nace la idea de concentrar pequeñas escuelas que se esparcen por el territorio en una escuela con internado que permita al alumnado desarrollar sus tareas en forma normal y continua.

El proyecto se establece en un llano del Valle de Pencahue, en la localidad de Botalcura, en el lugar en que se emplazaba la cancha de fútbol de una pequeña escuela de adobe.
Se desarrollaron dos patios, uno de los cuales alberga la actividad diurna (aulas) y el otro la actividad nocturna (dormitorios), complementados por volúmenes de servicios tales como, gimnasio, biblioteca, administración, baños, etc., con una superficie total de $4.300 \mathrm{~m}^{2}$.

Como primera etapa se construyeron $850 \mathrm{~m}^{2}$, con tres módulos destinados a aulas y dos a dormitorios. La modulación es de 3,84 m.; a ejes, entre contrafuertes. Se aplicó el criterio de mantener los contrafuertes hacia el exterior, de modo de evitar obstrucciones en el interior de las aulas. Dado que las condiciones de diseño en adobe no permitian llegar a un nivel de luminosidad natural aceptable, se optó por diseñar una cercha que entregará luz cenital a las aulas. La estructura se basa en aplicar criterios de simetria de tal forma de disminuir el riesgo de torsiones, se refor-

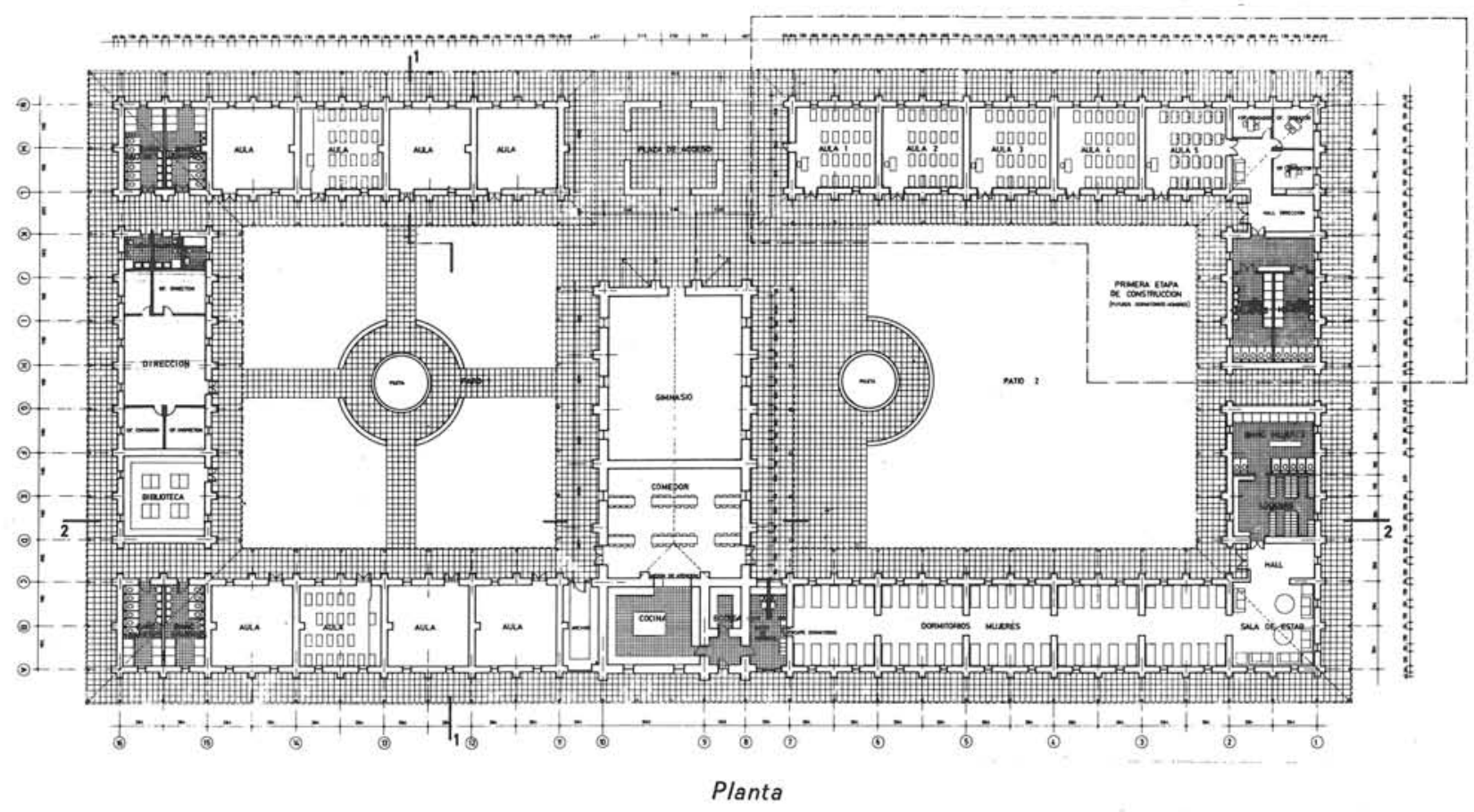


zaron las esquinas en forma de cruz y en los intermedios mediante un sistema de traba en te. Se diseñaron corredores exteriores e interiores en los pabellones, de' tal forma de disminuir el riesgo de daños ocasionados por la exposición de los muros de adobes a las aguas lluvias.

Se escogió el sistema de muros de albañileria.de adobe, tipo cabeza, de $10 \times 30 \times 58 \mathrm{~cm}$., por la seguridad que éste representaba, en el caso de solicitaciones sismicas. La legislación constructiva chilena, no permite el uso de adobe en edificios de uso público. Dadas las condiciones tecnológicas y de accesibilidad dificil del emplazamiento, se superó esta norma y se definió el sis- tema constructivo en adobe como el más apropiado.

El diseño en general, busca simplificar los elementos estructurales que pueden constituir un peligro en caso de sismo, es asi como se eliminaron los rellenos de dinteles, los que sin participar de la estructura, y de acuerdo a lo revisado en otros casos, actúan de forma contraproducente en caso de sismo.

La estructura de techumbre es de madera de pino y cubierta de plancha ondulada de fierro galvanizado, la que se colocó debido a la rapidez de montaje y facilidad de traslado desde el centro de abastecimiento.
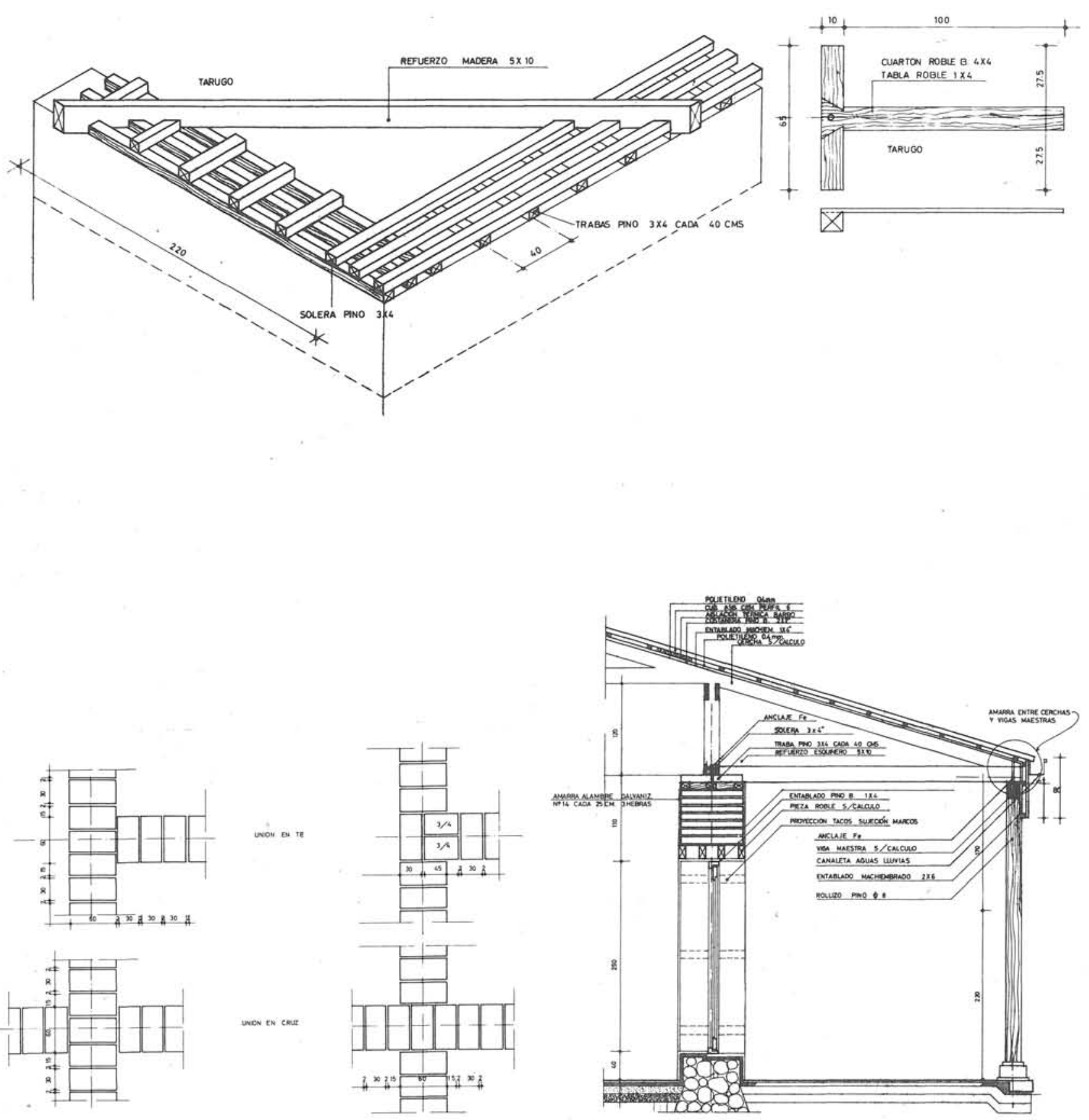

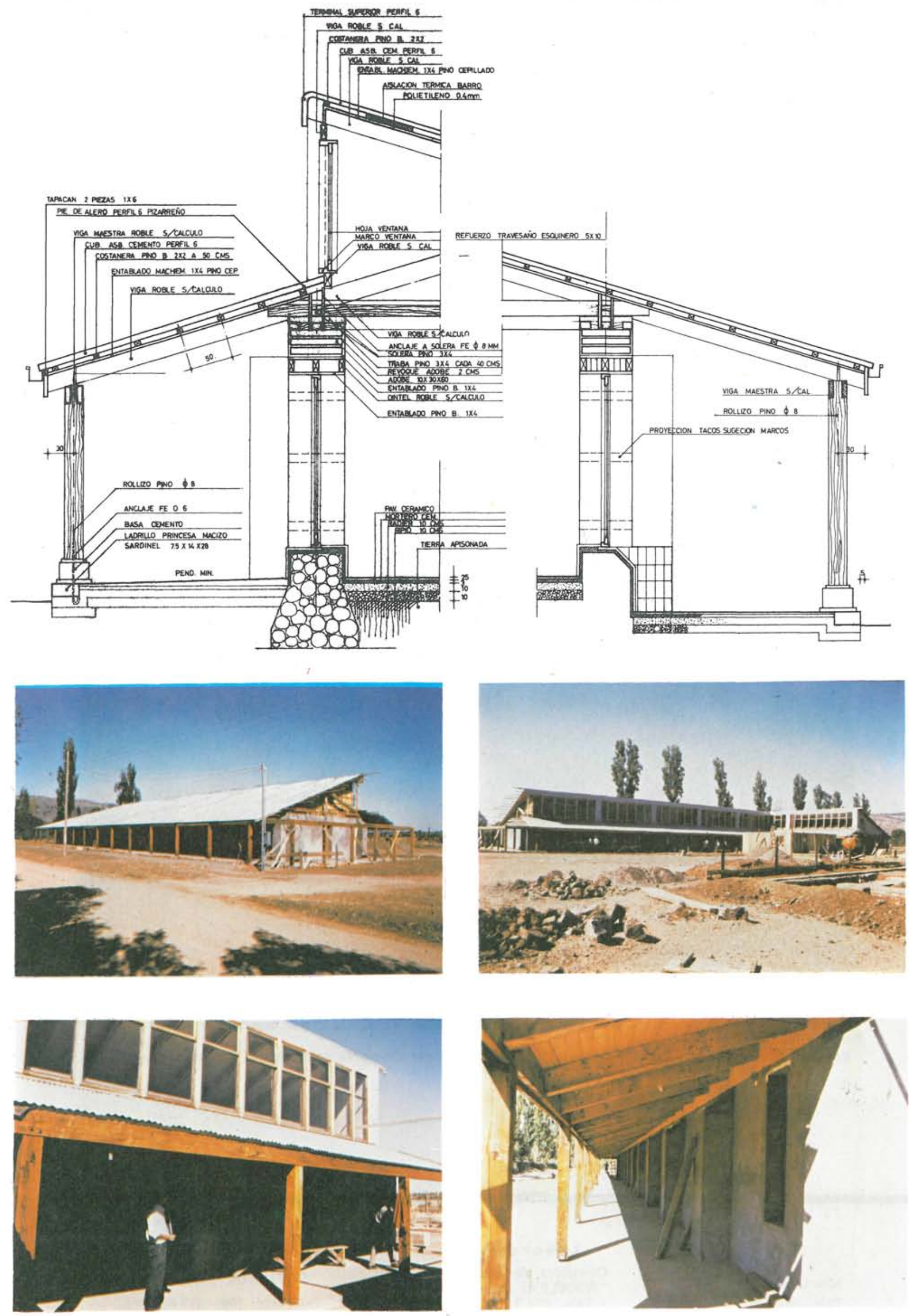